

\title{
Calibration of Single-axis Nanopositioning cell subjected to thermal disturbance.
}

Ning Tan, Cédric Clévy, Nicolas Chaillet

\section{To cite this version:}

Ning Tan, Cédric Clévy, Nicolas Chaillet. Calibration of Single-axis Nanopositioning cell subjected to thermal disturbance.. IEEE International Conference on Robotics and Automation, ICRA'13., Jan 2013, Germany. pp.3660-3665. hal-00876266

\section{HAL Id: hal-00876266 https://hal.science/hal-00876266}

Submitted on 24 Oct 2013

HAL is a multi-disciplinary open access archive for the deposit and dissemination of scientific research documents, whether they are published or not. The documents may come from teaching and research institutions in France or abroad, or from public or private research centers.
L'archive ouverte pluridisciplinaire HAL, est destinée au dépôt et à la diffusion de documents scientifiques de niveau recherche, publiés ou non, émanant des établissements d'enseignement et de recherche français ou étrangers, des laboratoires publics ou privés. 


\title{
Calibration of Single-axis Nanopositioning Cell Subjected to Thermal Disturbance
}

\author{
Ning Tan, Cédric Clévy, and Nicolas Chaillet
}

\begin{abstract}
In micromanipulation, especially microassembly, accuracy is a criterion useful for characterizing the performance of microrobots. The increase of positioning accuracy is a very important issue before making automatic assembly or other micro-tasks such as characterization. Thermal drift is one of the major sources of inaccuracy for automatic micromanipulation in ambient conditions and even in clean room. This paper addresses the calibration of a 1-DOF (Degree Of Freedom) nanopositioning cell including thermal drift compensation. The nanopositioning cell consists of a single-axis PZT stage and a XYZ manual stage which is usually used for fine positioning in microassembly platform. Subsequently, validations are implemented to test the performance by integrating the calibrated model. The experimental results show an effective improvement of the accuracy by a factor of 7 when temperature changes in the range of temperature for training, and 2.4 times improvement is achieved when temperature goes out of the training range.
\end{abstract}

\section{INTRODUCTION}

Micromanipulation (for example, microassembly) requires microrobots with high positioning accuracy to perform tasks at a high speed [1], [2]. To do this, we need to have reliable knowledge about the relative positions between the endeffector of micromanipulator and targets. Micromanipulators embedded with closed-loop controllers have high repeatability, but are unable to identify the aforementioned relationship by themselves. To remedy this problem, we can use either closed-loop sensory feedback control or calibration (in a broad point of view is open-loop control) [3]. The former could be accomplished via a microscope or/and a force sensor integrated at the microscale. However, there are some drawbacks. First, the exteroceptive sensors are generally bulky and often offer only one or two directions of measurement. Multi-direction of measurement requires to combine several sensors, which is a tough task because of the limited workspace [4]. Moreover, the time and computation cost paid is considerable. For example by visual servo control, image acquisition, feature extraction and other image processing (e.g., subpixel enhancement) introduce non-negligible time delays resulting in low assembly throughput. Last but not least, complex settings are required to make it work. On the other hand, calibration is a process of locating the endeffector of the manipulator in a global coordinate frame with acceptable or improved absolute accuracy by identifying and compensating errors in the control model. Thus open-loop

N. Tan, C. Clévy and N. Chaillet are with FEMTO-ST Institute, UMR CNRS-6174 / UFC / ENSMM / UTBM Automatic Control and Micro-Mechatronic Systems department (AS2M department), 24, rue Alain Savary, 25000 Besançon, France. ning.tan, cclevy, nicolas.chailletefemto-st.fr control after calibration is a rational alternative to improve efficiency and accuracy for micromanipulation [5].

Temperature is a major cause affecting positioning accuracy. In the condition of indoor temperature, drift of the nanopositioning stage can reach a few microns, which is not acceptable in many applications [6]. For example, the thermal drift of a $5 \mathrm{~cm}$ long aluminium bar can reach $1.2 \mu \mathrm{m}$ while temperature changes $1{ }^{\circ} \mathrm{C}$. However, microrobots should guarantee accuracy within submicron range when operating in uncontrolled environment where complex and nonlinear disturbances occur from everything (robot, measuring instruments, nearby devices, ...). One solution to wipe off temperature influence is using thermal-isolation or -stabilization equipments to keep temperature stable within the workspace. However, appropriate devices are not easy to get in the context of micro and nanoscale. Even in clean room (an environment more steady than normal labs), temperature variation is sufficiently large ( \pm 1 to $\pm 2{ }^{\circ} \mathrm{C}$ ) to affect the reference position and induce inaccuracy in micrometer range. Hence, our objective is to achieve submicron accuracy in a temperature changing environment.

There has been a lot of works on real-time thermal compensation. [7] applied and compared multiple regression analysis and artificial neural network for calibration of machine tools. An approach based on Gaussian integration technique was proposed for modeling thermally induced errors and selecting optimum temperature locations [8]. [9] used principal component analysis to find the temperature vector containing most information of the thermal-induced errors, and established thermal error models using orthogonal regression methods. In [10], thermal modal analysis was utilized for the temperature sensor placement determination and robust thermal error modeling for $\mathrm{CNC}$ machine tools. Zhao et al. [11] simulated the thermal deformation of a CNC spindle and calculated the thermal error using finite element method. Fraser et al. [12] developed expressions for the generalized transfer functions of the thermal, and thermal deformation response of the machine tool structure. Basically, these works considered large-sized and macropositioning robots or machine tools. However, due to the scale effects, thermal effects and induced-behavior at the microscale are different from those at the macroscale [1], [4], [13], [14]. A ultra-high-precision linear axis was calibrated while thermal effects acting on it in [15], [16]. Also about linear axis, we investigate the implementation of the model, performance after calibration and provide more discussions about modeling and experimental result. Some pioneers worked on calibration of micromanipulators in microassembly systems 
by deriving a mapping [3], [6] through interpolating a set of taught locations by least squares fit, but did not consider thermal errors.

To push forward the research in this domain, we investigate the calibration of a nanopositioning cell including a PZT stage (mobile stage) and a XYZ manual stage (static stage) taking into account thermal drift. What are new in this paper are the discussions of modeling foundation, physical meaning, temperature selection in Section II, and the validation of the positioning performances after calibration in Section III.

\section{MODELING AND IDENTIFICATION}

There are two main set of methods for thermal-drift modeling [17], which are principle-based methods and empiricalbased methods. The heat transfer model of the compact and small-sized system is very complex which is difficult to be built analytically if is not impossible. So we used the latter due to the fact that empirical-based method is more suitable for modeling the relation between temperature variation and part deformation. Here we investigate a static model based on the quasi-static assumption that thermal errors vary slowly with time and are only related to the mechanical structure. The geometric errors of the mobile stage are required to model independently to thermal drift modeling.

\section{A. Geometric modeling}

For the sake of convenience, we investigate inverse kinematic modeling directly instead of first forward kinematics and then inverse kinematics. The following model is chosen:

$$
q_{g}=a_{1} x^{n 1}+a_{2} x^{n 1-1}+\ldots+a_{n 1} x
$$

where $x$ is the measured position by external sensor; $q_{g}$ is the control input which is usually the motors coordinates of the robot; $a_{1}, \ldots, a_{n 1}$ are geometric coefficients; $n 1$ is the order geometric model. Depending on different nanopositioning stages, the best order can be decided by comparing the fitting errors. The order with smallest fitting error is selected.

\section{B. Thermal-Drift Modeling}

1) Thermal-drift measurement: Except for geometric errors, the system is also highly susceptible to thermal disturbances. For instance, we use PID control to keep a PZT stage at its zero position with internal capacitance sensor. An interferometer is used as external sensor measuring the real position of the stage. As seen in Fig. 1, there is drift up to $0.4 \mu \mathrm{m}$ when temperature decreases $0.35{ }^{\circ} \mathrm{C}$. Meanwhile, internal sensor indicates the controller achieves to control the stage at zero position (with $100 \mathrm{~nm}$ measuring noises). From Fig. 1(d), we can see input voltage (also the output of the PID controller) of the stage changes during this time, which means the internal sensor detect a part of drift and the controller compensate it. However, internal sensor misses the part detected by interferometer because of the location of internal sensor. Therefore calibration is required to compensate the remaining drift. To choose a suitable calibration model, we first perform an experiment to characterize the relation
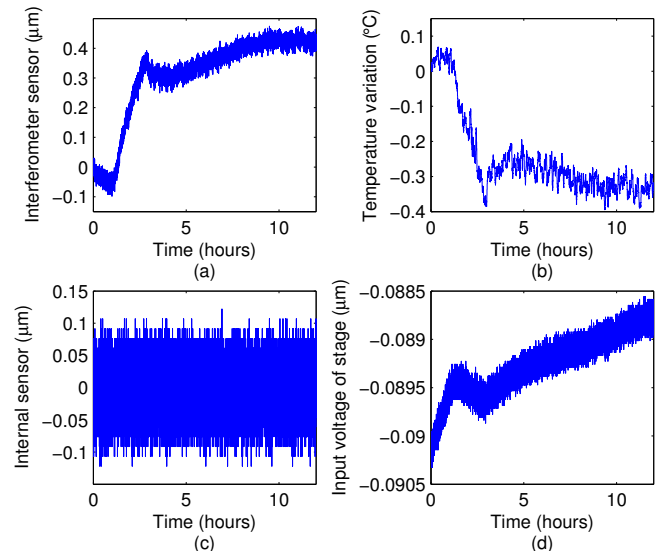

Fig. 1. (a) Measurement of stage position by interferometer; (b) Measurement of ambient temperature close to the stage; (c) Measurement of internal sensor of the stage; (d) Input voltage of the stage.

between temperature and thermal drift. In this experiment, the interferometer is used to measure the position of the switch-off PZT stage. The interferometer is defined as global frame in two days of measurement. Even though without inputting moving commands, the interferometer detects the drift of the stage. From Fig. 2, it can be seen that there is drift increasing with the temperature decreasing in an opposite way. These figures show a roughly linear relation between temperature variation and position drift. It is worth noting that the drift measured including not only the drift of the stage, but also the drift of interferometer which we try to minimize.
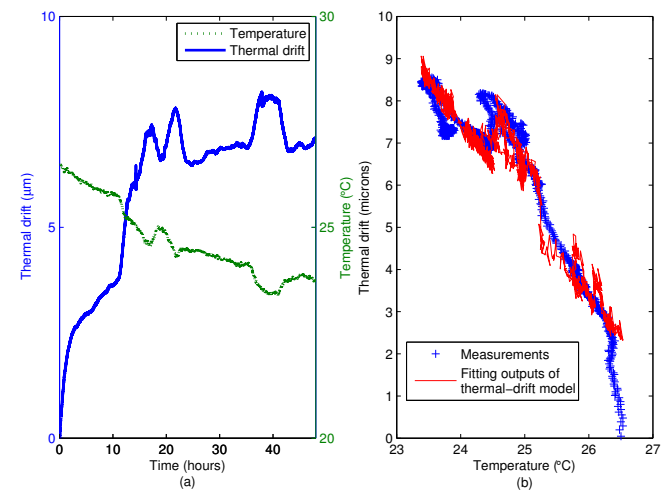

Fig. 2. Relationship between temperature and thermal drift:(a)Temperature change and thermal drift with time; (b)Measurement and model fitting of thermal drift.

2) Modeling: The drift is mainly due to the thermal elongation $\delta$ in different parts of the stage. Considering a ideal and simple case, the elongation $\delta$ can be computed based on the following equation:

$$
\delta=L-L_{0}=L \cdot \alpha \cdot\left(T-T_{0}\right),
$$

where $L_{0}$ is the length at reference temperature $T_{0}, \alpha$ is thermal-expansion coefficient, $L$ is the length of component 
after elongation, and $\delta$ is the drift to be compensated. The whole thermal drift of the nanopositioning cell is composed of thermal-expansion of different parts. Therefore, the $1 \mathrm{st}$ order relationship between the temperature and the drift is the modeling foundation of this paper.

Moreover, considering the nonuniform temperature field and nonlinear combination of thermal expansions, we monitor temperatures at several points of the workspace and have the thermal-drift model:

$$
q_{t}=b_{1} t_{1}+\ldots+b_{n 2} t_{n 2}+\gamma
$$

where $q_{t}$ is thermal compensation input; $t_{1}, \ldots, t_{n 2}$ are measured temperatures; $b_{1}, \ldots, b_{n 2}$ are temperature coefficients; $\gamma$ plays a role of bias; $n 2$ is the order thermal-drift model. The model correlates temperature field to corresponding induced drift through thermal coefficients $b_{1}, \ldots, b_{n 2}$. Using this model we can approximate the thermal drift with temperature information, and the fitting result is shown in Fig. 2 (b).

To determine the suitable model order, a comparison of the 1st-order and 2nd-order is shown in Fig. 3 of which first part is the training phase, the second than third parts are compensation phases. The closer the compensation curves to the drift curves is better. We can see that the compensation of the 1st-order model is closer to the drift than the 2nd-order. It means that the 1st-order has better performance which will be used for calibration.

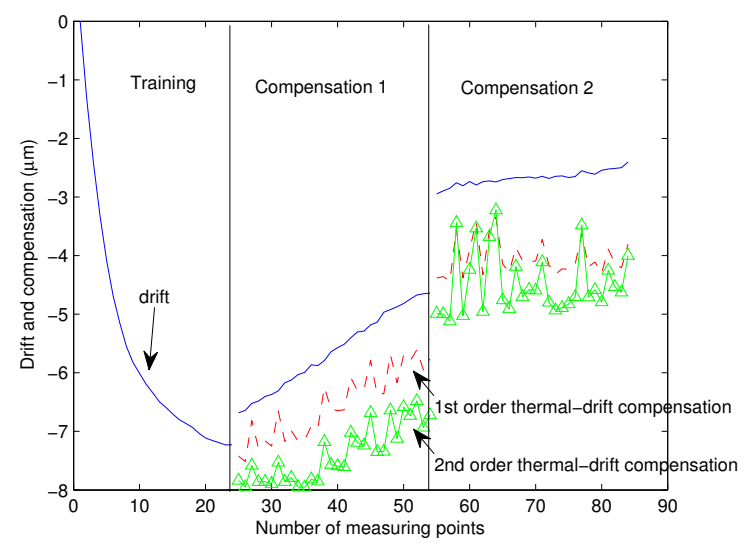

Fig. 3. Comparison of compensation effects of the 1st and the 2nd order thermal-drift models.

3) Temperatures selection: Because there are several temperatures in the model and not all of them are useful for approximating the thermal drift, temperatures should be selected to form a best model. The identification algorithm we use is stepwise algorithm which is able to choose suitable temperature to fit the model automatically. However, the temperature selection should be aided sometimes by our experience or observation. For example, in a experiment, a hot plate is used to heat the nanopositioning cell up. A thermocouple is placed on the hot plate to measure its temperature which is also included in the training data of the model. After training, the algorithm keeps the hot plate temperature for the final model. However, from Fig. 4, we can see the compensation effect excluding hot-plate temperature is superior than that including hot-plate temperature. This is because that the temperature rising speed of the hotplate is much faster than that of the air which affects the cell by convection.

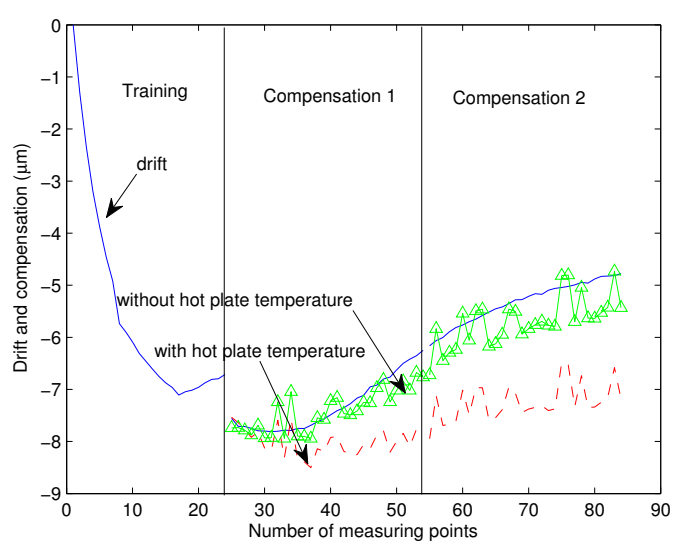

Fig. 4. Comparison of compensation effects with and without taking into account hot-plate temperature.

\section{Complete Model and Identification}

Combining geometric model and thermal drift model yields the complete model:

$$
q=a_{1} x^{n 1}+a_{2} x^{n 1-1}+\ldots+a_{n 1} x+b_{1} t_{1}+\ldots+b_{n 2} t_{n 2}+\gamma
$$

This model is chosen because it has efficient performance, simple and straightforward physical meaning which help to know better the behavior, and to exploit a more powerful model in the future.

Obtaining high fitting precision (submicrons) requires a set of measuring data for training:

$$
\left[\begin{array}{c}
q_{1} \\
\vdots \\
q_{m}
\end{array}\right]=\sum_{i=1}^{n 1} a_{i}\left[\begin{array}{c}
x_{1}^{n 1-i+1} \\
\vdots \\
x_{m}^{n 1-i+1}
\end{array}\right]+\sum_{i=1}^{n 2} b_{i}\left[\begin{array}{c}
t_{i, 1} \\
\vdots \\
t_{i, m}
\end{array}\right]+\gamma
$$

where $m$ is the number of the measurements.

The equation could be written as matrix form

$$
\left[\begin{array}{c}
q_{1} \\
\vdots \\
q_{m}
\end{array}\right]=\left[\begin{array}{ccccccc}
x_{1}^{n 1} & x_{1}^{n 1-1} & \ldots & x_{1} & t_{1,1} & \ldots & t_{n 2,1} \\
\vdots & \vdots & \vdots & \vdots & \vdots & \vdots & \vdots \\
x_{m}^{n 1} & x_{m}^{n 1-1} & \ldots & x_{m} & t_{1, m} & \ldots & t_{n 2, m}
\end{array}\right]\left[\begin{array}{c}
a_{1} \\
\vdots \\
a_{n 1} \\
b_{1} \\
\vdots \\
b_{n 2}
\end{array}\right]+\gamma
$$

In the case study of this paper, the fitting error of the 3rd order geometric model is smallest and four thermocouples are used. To perform training and parameters identification, the stepwise regression (Matlab ${ }^{\circledR}$, StatisticsToolbox ${ }^{T M}$ ) 
is used because it is able to automatically search the coefficients space and keeps the most influential ones by calculating the p-value of F-statistic. The algorithm could be implemented conveniently by a Matlab function stepwisefit.

\section{A CASE STUDY}

\section{A. Experimental Setup}

As shown in Fig. 5, the system of the case study consists of a 1-DOF PZT stage P-625.1CD (Physik Instrumente) with the resolution of $1.4 \mathrm{~nm}$, a XYZ manual stage, a laser interferometer (SP-S 120 SIOS Meßtechnik GmbH) with the resolution of $0.3 \mathrm{~nm}$, and four $\mathrm{K}$ type thermocouples. The PZT stage with internal sensor in closed-loop mode (with PID control) has positioning accuracy of $300 \mathrm{~nm}$ tested in a temperature stabilized environment (with \pm 0.05 ${ }^{\circ} \mathrm{C}$ stability). The interferometer defines the global frame during training and validation by setting the initial reading at the beginning of training phase as zero. According to [18], the three main measuring uncertainties of the interferometer system come from the wavelength compensation, deadpath correction and material thermal compensation. Herein, wavelength compensation and deadpath correction are realized by built-in temperature/pressure-correction module (incorporates a temperature sensor Pt100 and barometric-pressure sensor as seen in Fig. 5). According to the calculation method in GUM (Guide to the Expression of Uncertainty in Measurement) [18], we calculate the measurement uncertainty of the interferometer less than $40 \mathrm{~nm}$ taking into account intrinsic and extrinsic factors. T1 is glued on the PZT stage. T2 measures the temperature of the bracket connecting PZT stage and XYZ manual stage. T3 and T4 monitor the air temperature around the workspace. Serval thermocouples are required because the modeling of thermal drift in this work must be precise but there are temperature gradients in the workspace and the temperatures at different locations are not exactly the same. In addition, the experimental setup is covered by a shield against air flow. The PZT stage is mobile stage and the XYZ manual stage is static stage. Both stages generate thermal drift, but only PZT stage could be controlled to compensate the combined drift of the cell.

\section{B. Implementation of Calibration}

The implementation of calibration is according to the flowchart in Fig. 6. The workspace of the PZT stage is a line of $500 \mu \mathrm{m}$. The measurements of motor coordinates $q$ are taken at 0306090120150180210240270300330360 $390420450480 \mu \mathrm{m}$ in forward motion phase. The control trajectory is shown in Fig. 7 which demonstrates that, in every cycle, the rising time of the input signal is 4 seconds and the input keeps constant 25 seconds before the next. The average values in the last 5 seconds of every step are considered as the measurements $x$ and used for training. Four thermocouples obtain a set of temperature data $t$. It takes about 4 hours for 24 cycles data acquisition. All the data are fed into Stepwise algorithm for training. The identified parameters $C$ are embedded into the controller.
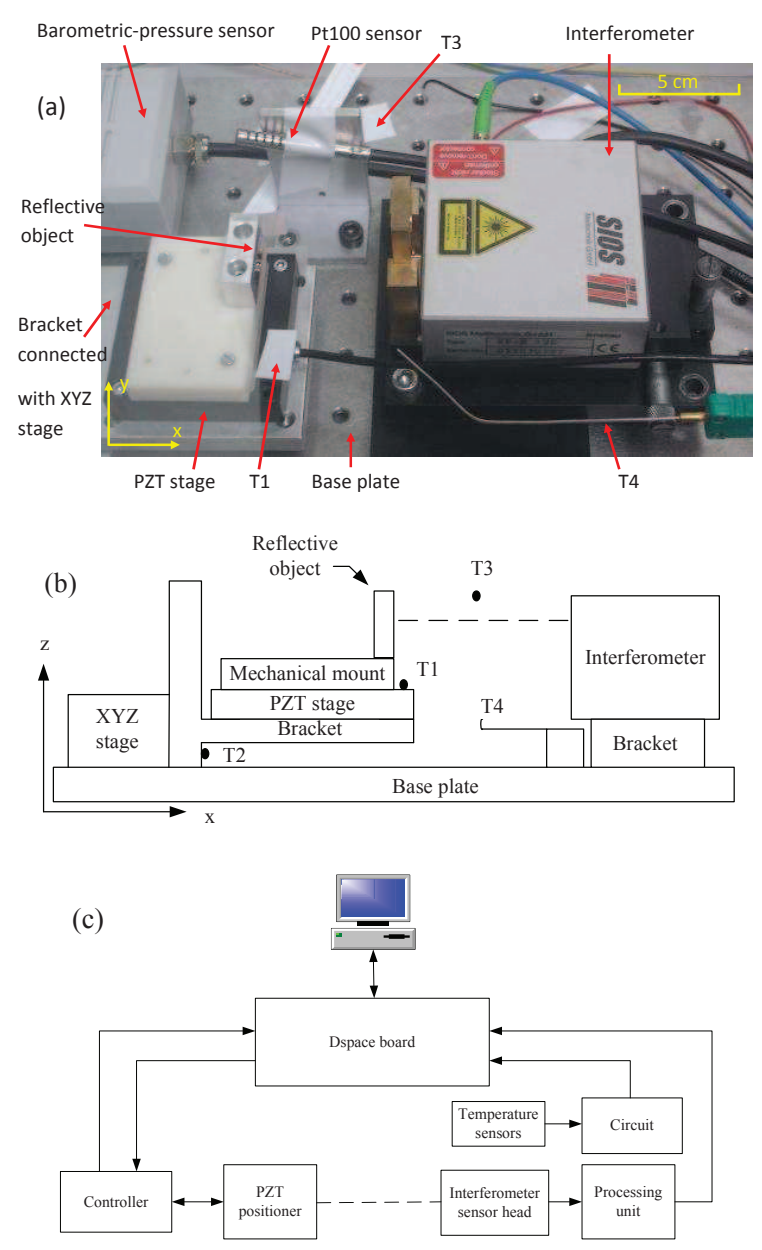

Fig. 5. Experimental setup comprising 1-DOF PZT stage and reflective object, interferometer with affiliated pressure/temperature sensors, four temperature sensors: (a) Photograph of the PZT stage and measuring system; (b) Side view of placement of four temperature sensors $\mathrm{T} 1 \rightarrow \mathrm{T} 4$; (c) Diagram of the hardware allocation.

Afterwards, adapting the calculation method of ISO-9283 [19] into 1-DOF, validations are conducted to test the performances after calibration. The test points $x^{\prime}$ for validation are taken at coordinates $080160240320400480 \mu m$. These points include the ones (0 240480$)$ also considered in training and the ones (80 160320400 ) never used for training. Validating the points both inside and outside the training set could help to evaluate performance of both robustness and generalization (or interpolation). Every test of validation takes 1.5 hours for 30 cycles. New motor coordinates $q^{\prime}$ are obtained by the model and fed to control the stage to positions $x^{\prime \prime}$ measuring by the interferometer.

\section{Results and Discussions}

If the input data is substituted into the model calculated with the coefficients, we have the differences between every fitting (calculated) component and the target as well as the positioning error shown in Fig. 8. The discrepancy between the real (measured) position $x$ and the target $q$ yields the positioning error. The geometric model error is the difference between the fitting output $q_{g}$ of the geometric model (1) 


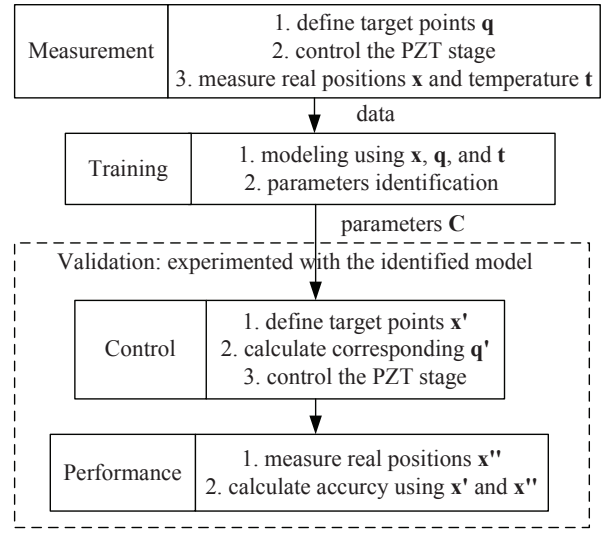

Fig. 6. Flow chart of the calibration procedure.

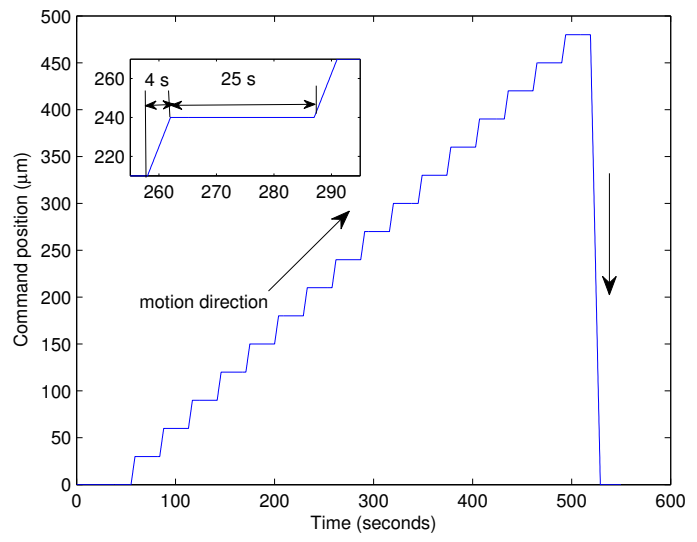

Fig. 7. Command trajectory of measurement for 1 cycle.

and the target $q$. The thermal-drift compensation is the fitting output $q_{t}$ in (2). Final fitting error is the residual difference between the fitting output of the whole model $q_{g}+q_{t}$ and the target $q$. From this figure, we can see that $q_{g}-q$ deviates from zero because geometric model does not take into account thermal drift. So $q_{t}$ deviates in opposite direction to compensate $q_{g}-q$. Finally the fitting errors are drawn back to the area around zero.

In validation phase, five tests are performed. The first is a preliminary test without using calibrated model to control the PZT stage; from the second to the fourth, the model uses well-trained model to control the stage. The compensation effects and corresponding ambient temperature (measuring by T4) at zero position (the target point of the PZT stage is zero) are shown in Fig. 9 and 10. It is clear the compensation curves from the test 2 to test 4 approximate the drift curves very well, which means that they compensate most of the drift. Test 5 still compensates most of the drift even though the drift has become nearly 3 times of that in calibration. Accuracy results (absolute errors between mean measured values and targets) with and without compensation are shown in Fig. 11 and Table I. Because from test 2 to test 5 the compensation is running, the accuracy values without calibration are obtained by additional calculation in order to compare

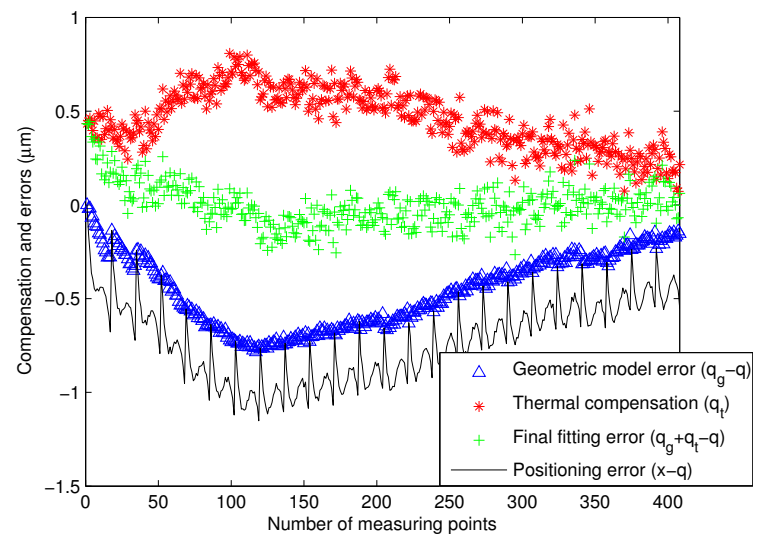

Fig. 8. Positioning error and effect of the model. Geometric model error: $q_{g}-q$; Thermal compensation: $q_{t}$; Final fitting error: $q_{g}+q_{t}-q$; Positioning error: $x-q$.

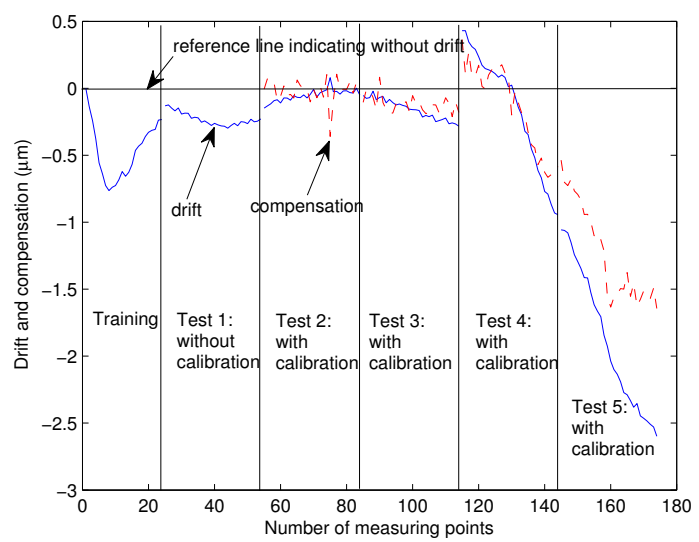

Fig. 9. Experimental results of drift and compensation at zero positions during all experiments.

the performances with and without compensation during the same period of time. There are 7 to 10 times enhancement of accuracy achieved in test 2, 3, and 4. And test 5 also improves by 2.4 times after calibration more than two hours. In Fig. 10, it can be seen that the ambient temperature variation of test 5 increases nearly by $0.7{ }^{\circ} \mathrm{C}$ which is far away from the range of temperature in training phase. Hence, the proposed calibration method effectively enhances the positioning accuracy of the nanopositioning cell, and is reliable when temperature changes to values far from the interval used for calibration. To obtain the best performance and robustness, the range of training temperature should be wide enough and include increasing and decreasing phases.

\section{CONCLUSIONS AND FUTURE WORKS}

Thermal drift is a major cause of inaccuracy in micro and nanomanipulation (from Table I, we can see the accuracies from the test 2 to the test 4 are about $0.5 \mu \mathrm{m}$ and in test 5 more than $2 \mu \mathrm{m}$ suffering temperature change). Especially in microassembly, thermal drift can degrade positioning accuracy and effective throughput. This paper proposes a 
TABLE I

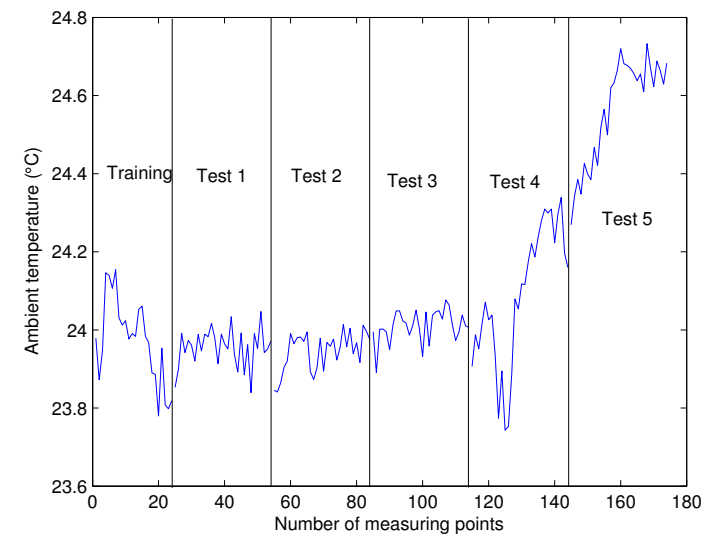

Fig. 10. Ambient temperature (measuring by T4) evolution at zero positions during all experiments.

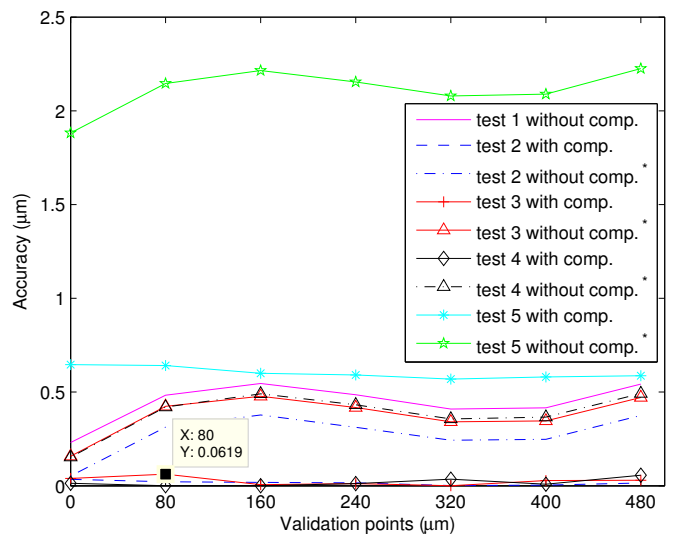

Fig. 11. Experimental results of accuracy with and without compensation. * from calculation but not measurement.

full scheme of calibration for a 1-DOF nanopositioning cell subjected to thermal disturbance. Based on measurements of interferometer and thermocouples, a set of experiments has been conducted to characterize, improve, and validate the performances of the system. Experimental results show that the submicron accuracy is realized. The accuracy is improved by nearly 7 to 10 times in the first four tests when temperature changes within or close to the range of training. These results are even better than the accuracy $(300 \mathrm{~nm})$ of PZT stage without calibration in steady temperature, and are close to the measurement uncertainty $(40 \mathrm{~nm})$ of the interferometer which means they are the best results we can achieve based on existing devices. When temperature exceeds the range much in test 5 , accuracy is still improved by 2.4 times. In the future, efforts will be conducted towards multi-DOF microrobots calibration considering thermal drift. Moreover, a superior model would be refined and the robustness of the calibration will be investigated.

\section{ACKNOWLEDGMENTS}

This work has partially been supported by the FrancheComté region and OSEO. The authors would like to thank
COMPARISON OF ACCURACY WITH AND WITHOUT COMPENSATION

\begin{tabular}{|c||c|c|c|}
\hline Test & Without comp. & With comp. & Percentage of Improvement \\
\hline 1 & $546 \mathrm{~nm}$ & $\backslash$ & $\backslash$ \\
\hline 2 & $378 \mathrm{~nm}^{*}$ & $35 \mathrm{~nm}$ & $980 \%$ \\
\hline 3 & $478 \mathrm{~nm}^{*}$ & $62 \mathrm{~nm}$ & $671 \%$ \\
\hline 4 & $492 \mathrm{~nm}^{*}$ & $57 \mathrm{~nm}$ & $763 \%$ \\
\hline 5 & $2225 \mathrm{~nm}^{*}$ & $646 \mathrm{~nm}$ & $244 \%$ \\
\hline
\end{tabular}

* from calculation but not measurement.

David GUIBERT for fabrication of mechanical parts.

\section{REFERENCES}

[1] N. Chaillet and S. Régnier, Microrobotics for Micromanipulation, Wiley-ISTE; 2010

[2] K. Rabenorosoa, C. Clévy, Q. Chen, and P. Lutz, Study of Forces during Micro-Assembly Tasks using Two-Sensing-Fingers Gripper, IEEE/ASME T MECH, vol. 17, no. 5, 2012, pp 811-821.

[3] A.N. Das, R. Murthy, D.O. Popa, and H.E. Stephanou, A Multiscale Assembly and Packaging System for Manufacturing of Complex Micro-Nano Devices, IEEE T AUTOM SCI ENG, vol. 9, no. 1, 2012, pp $160-170$

[4] C. Clévy, M. Rakotondrabe, and N. Chaillet, Signal Measurement and Estimation Techniques for Micro and Nanotechnology, Springer; 2011.

[5] M. Rakotondrabe, C. Clévy, and P. Lutz, Complete open loop control of hysteretic, creeped and oscillating piezoelectric cantilever, IEEE T AUTOM SCI ENG, vol. 7, no. 3, 2009, pp 440-450.

[6] L. S. Mattos and D. G. Caldwell, A Fast and Precise Micropipette Positioning System Based on Continuous Camera-Robot Recalibration and Visual Servoing, in Pro. IEEE CASE, 2009, pp 609-614.

[7] J.S. Chen, J. Yuan, and J. Ni, Thermal Error Modelling for Real-Time Error Compensation, INT J ADV MANUF TECH, vol. 12, 1996, pp 266-275.

[8] D.A. Krulewich, Temperature Integration Model and Measurement Point Selection for Thermally Induced Machine Tool Errors, Mechatronics, vol. 8, 1998, pp 395-412.

[9] C. Gong, J. Yuan, and J. Ni, Nongeometric Error Identification and Compensation for Robotic System by Inverse Calibration, INT $J$ MACH TOOL MANU, vol. 40, 2000, pp 2119-2137.

[10] J. Zhu, Robust Thermal Error Modeling and Compensation for CNC Machine Tools, PhD Thesis, The University of Michigan, 2008.

[11] H.T. Zhao, J.G. Yang, and J.H. Shen, Simulation of Thermal Behavior of a CNC Machine Tool Spindle, INT J MACH TOOL MANU, vol. 47, 2007, pp 1003-1010.

[12] S. Fraser, M.H. Attia, and M.O.M. Osman, Modelling, Identification and Control of Thermal Deformation of Machine Tool Structures, Part 2: Generalized Transfer Functions, J MANUF SCI ENG, vol. 120, 1998, pp 632-639.

[13] K.S. Kim, J.C. Heo, and K.W. Kim, Effects of Temperature on the Microscale Adhesion Behavior of Thermoplastic Polymer Film, Tribology Letters, vol. 38, no. 2, 2010, pp 97-106.

[14] J.W. Li, G.S. Yang, W.J. Zhang, S.D. Tu, and X.B. Chen, Thermal Effect on piezoelectric stick-slip actuator systems, REV SCI INSTRUM, 79, 046108, 2008.

[15] E. Lubrano, Calibration of ultra-high-precision robots operating in an unsteady environment, PhD Thesis, No. 5098, Lausanne, EPFL, 2011.

[16] E. Lubrano and R. Clavel, Thermal Behavior of an Ultra HighPrecision Linear Axis Operating in Industrial Environment. 9th International Workshop on Research and Education in Mechatronics, 2008, pp 151-152.

[17] J.W. Li, W.J. Zhang, G.S. Yang, S.D. Tu, and X.B. Chen, ThermalError Modeling for Complex Physical Systems: The-State-of-the-Arts Review, INT J ADV MANUF TECH, 2009.

[18] H. Castro, Uncertainty Analysis of a Laser Calibration System for Evaluating the Positioning Accuracy of a Numerically Controlled Axis of Coordinate Measuring Machines and Machine Tools, PRECIS ENG, vol. 32, no. 2, 2008, pp 106-113.

[19] ISO 9283:1998, Manipulating Industrial Robots Performance Criteria and Related Test Methods, 1998. 